\title{
Suspected penile fracture: to operate or not to operate?
}

\author{
Ian S. Metzler, Amanda B. Reed-Maldonado, Tom F. Lue \\ Department of Urology, University of California at San Francisco, San Francisco, CA, USA \\ Correspondence to: Tom F. Lue, MD, ScD (Hon), FACS. 400 Parnassus Ave, Suite A610, San Francisco, CA 94143-0738, USA. Email: Tom.Lue@ucsf.edu.
}

Submitted Jun 13, 2017. Accepted for publication Jul 13, 2017.

doi: $10.21037 /$ tau.2017.07.25

View this article at: http://dx.doi.org/10.21037/tau.2017.07.25

Distinguishing penile ecchymosis from a true penile fracture, or a rupture through the tunical albuginea, can be a challenging clinical conundrum. A penile fracture typically occurs in the setting of blunt penile trauma to the erect penis, most often during sexual intercourse or masturbation. The characteristic symptoms of penile fracture are a "snapping" or "popping" sound, penile pain, and immediate detumescence followed by ecchymosis and swelling of the penile shaft (1). Physical examination findings may vary significantly in patients with a history suggestive of penile fracture, and the severity of the penile ecchymosis frequently does not correlate with the presence or absence of tunical rupture. History and physical examination may be inaccurate in $15 \%$ of patients with a suspected penile fracture (2). This clinical picture can be even more confusing if the patient received collagenase clostridium histolyticum $(\mathrm{CCH})$ in the days or weeks prior to presentation. In recent years, the increasing use of $\mathrm{CCH}$ for Peyronie's disease has resulted in an upsurge in the number of patients presenting to urgent care with a history suggestive of penile fracture and significant penile edema and ecchymosis.

Penile fracture is considered an urgent urologic condition that requires timely repair to prevent long-term complications of corporal fibrosis, penile curvature, chronic pain, and erectile dysfunction (3). Concomitant urethral injury occurs in $10-25 \%$ of cases and can result in bleeding from the urethra or voiding difficulty (4). If the fracture decompresses through the urethra, the findings on physical examination are typically relatively benign. Rapid operative repair of the corporal injury and urethral injury, if present, is associated with a lower risk of permanent erectile dysfunction and wound-healing complications (5). On the other hand, penile ecchymosis or hematoma secondary to rupture of the superficial vessels of the penis is not a surgical emergency and may be managed conservatively without any longterm detriment to the patient. Differentiating these clinical scenarios is critical to making the correct management decision, and physical examination alone may not be sufficient. Informed consent was obtained from all patients prior to publication of these case reports and accompanying images.

Figure 1 depicts the presentation, with impressive physical exam, of a 52-year-old-male with a history of Peyronie's disease and CCH injection 2 weeks prior to presentation. This patient has both a history convincing for penile fracture and an impressive physical exam with severe ecchymosis and edema of the penis. However, in the setting of $\mathrm{CCH}$ injection, he was also at high risk for superficial penile hematoma without corporal rupture. Penile ultrasound (US) performed at the bedside revealed a subcutaneous hematoma with an intact tunica albuginea (TA) and no intracavernous hematoma. Conservative treatment was recommended with loose compression dressings for 7 days and abstinence from intercourse until ecchymosis resolved. His ecchymosis was completely resolved and normal erections returned after 3 weeks.

To augment the diagnostic evaluation of severe penile ecchymosis and to avoid unnecessary penile surgery and the subsequent associated morbidity, various imaging modalities, such as magnetic resonance imaging (MRI), retrograde urethrography (RUG), cavernosography, and US have been utilized. MRI has high sensitivity for soft tissue and is effective in detecting disruption of the TA (6). However, cost, time, and availability are all obstacles to its use in the routine evaluation of penile trauma (7). RUG should be performed if there is any suspicion of urethral injury, and if positive, the patient should be taken for penile exploration and surgical repair. RUG is sensitive, low cost, familiar, and readily available. Nonetheless, it does not 

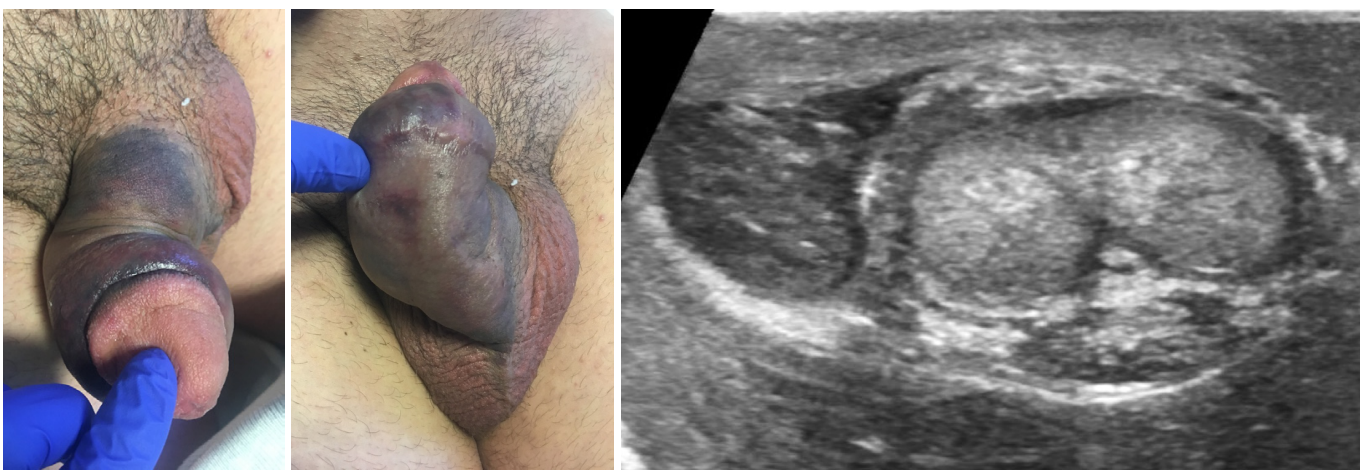

Figure 1 A 52-year-old-male with Peyronie's disease with a 90-degree dorsal curvature who have two cycles of CCH separated by three months. The patient had mild, transient penile ecchymosis after his first injection. During his first attempt at intercourse 2 weeks after his $2^{\text {nd }}$ injection, he felt a "pop", lost his erection, and noted a quickly expanding hematoma around his penis. He had no associated hematuria.

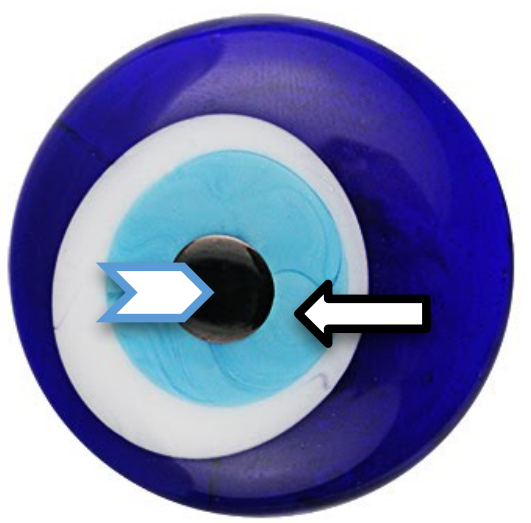

Figure 2 The tunica albuginea is homologous to the white circle of the Turkish eye symbol. The erectile tissue within the corpus cavernosum is homologous to the light blue area (arrow). The hematoma is homologous to black area (arrowhead).

provide information about corpora cavernosa injury in most cases. Cavernosography is invasive, time consuming, and plagued by false negatives (8). Penile US has been used in cases of penile fracture with variable reported success (9-11). Penile fracture is a rare entity, and radiologists do not routinely perform penile US, which may impede diagnostic certainty. Given these reservations, no specific imaging modality has been routinely utilized in cases of suspected penile fracture. Penile fracture often remains a clinical diagnosis that frequently involves surgical exploration to make the definitive diagnosis.

A growing body of evidence suggests that advancements in US technology over the last decade may make US increasingly more reliable in the diagnosis of penile fracture (12-15).
High-resolution gray-scale images and high-frequency broadband transducers with color Doppler capability allow for clear imaging of the TA, corpus cavernosa, and superficial penile vasculature $(15,16)$. Our institution relies on clinical examination and patient history to dictate the diagnosis and treatment of a penile fracture. When there is any doubt, we utilize penile US to aid in the evaluation and confirm the diagnosis. In patients with suspicion of fracture who have recently been treated with $\mathrm{CCH}$, we perform penile US for all patients. We propose that contemporary US, with its familiarity to urologists, ease-of-use, improved fidelity, availability in the office and the emergency room, and relative low cost, should be readily employed to diagnose penile fractures. Judicious use of penile US will avoid unnecessary diagnostic confusion, delay in treatment, excessive expense, and negative surgical exploration.

Some clinicians, radiologists and urologists alike, may contend that penile US is an unfamiliar diagnostic technique that they are uncomfortable employing. The penile US technique involves of imaging the entire penile shaft with a high-frequency (7-12.0 MHz linear transducer) transducer in both the transverse and horizontal planes to examine for defects in the TA. We propose that there are two penile US findings-discontinuity of the TA and/ or intracavernosal hematoma or the "Turkish eye sign" (Figure 2) - that conclusively diagnose penile fracture and that both urologists and radiologists can identify these signs on sonographic images with accuracy and confidence. In the absence of these two US findings (Figure 1), urologists may consider conservative management with loosely-applied compressive dressings and abstinence until the ecchymosis resolves completely. 

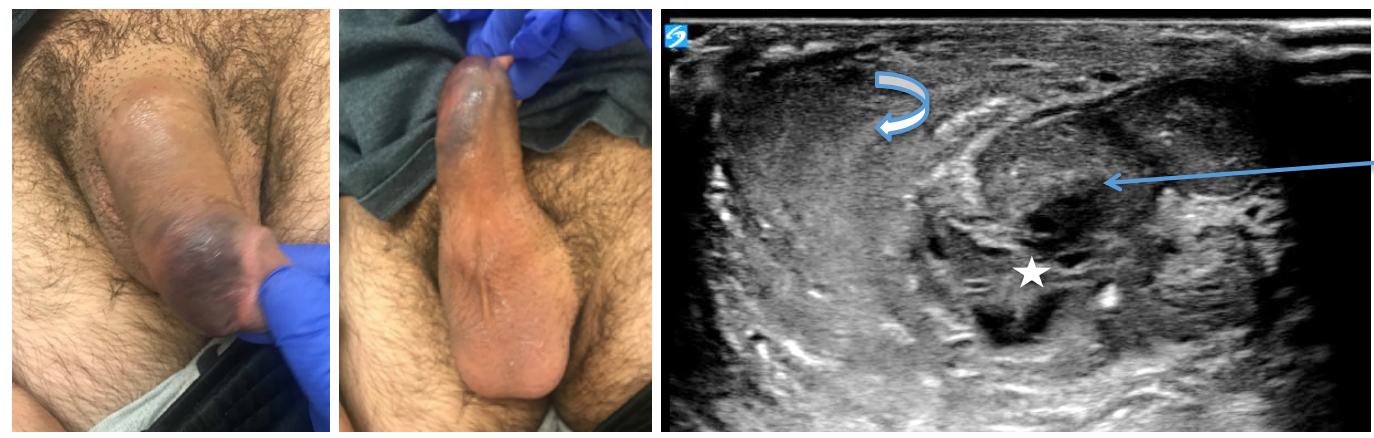

Intracavernous hematoma with "Turkish eye sign". (Arrow). Disruption of tunica albuginea (Star). Subcutaneous edema and hematoma (Curved arrow).

Figure 3 A 27-year-old male with a history of penile trauma self-inflicted while quickly pulling up his belted pants over an erection. He did not lose his erection immediately. He had no popping sensation and no hematuria. He was on no relevant medications, and has no PMH or PSH. He presented to the emergency room with mild distal shaft ecchymosis with minimal penile edema.

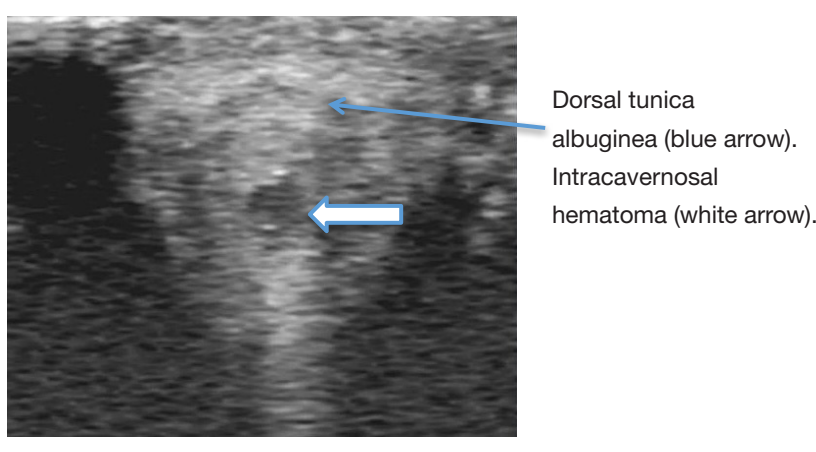

Figure 4 This is a 64-year-old man who reported a classic history for penile fracture with gross hematuria. Physical examination revealed mild, very distal penile edema and ecchymosis near the corona of the glans penis. Penile ultrasound performed from the dorsal aspect of the penis revealed an intracorporeal hematoma, or "Turkish eye sign" (white arrow). Surgical exploration revealed a 1 $\mathrm{cm}$ transverse laceration of the tunica albuginea of the distal corpora cavernosa along with a laceration of corpus spongiosum and urethra.
In many cases of suspected penile fracture, a clearly disrupted TA is not well visualized. This is likely because rupture exposes the collagen of the TA to blood, and the exposed collagen is one of the most powerful initiators of blood clotting in vivo. This exposure activates the clotting cascade and allows for rapid thrombosis, effectively sealing the TA and making a tunical tear difficult to visualize. In such cases, though, this same clotting cascade leads to the development of an intracavernosal hematoma, seen on US as a circular or irregular hypoechoic region surrounded by echo-dense corporal tissue (Figures 3-5). Figure 3 depicts a 27 -year-old male with self-inflicted penile trauma. This patient had an atypical presentation without the usual associated historical features such as a "pop", pain, or loss of erection. Bedside US of the penis showed subcutaneous hematoma, clear disruption of the TA border, and the intracorporal "Turkish eye sign" with irregularity of the normally homogenous corporal tissue. Penile exploration
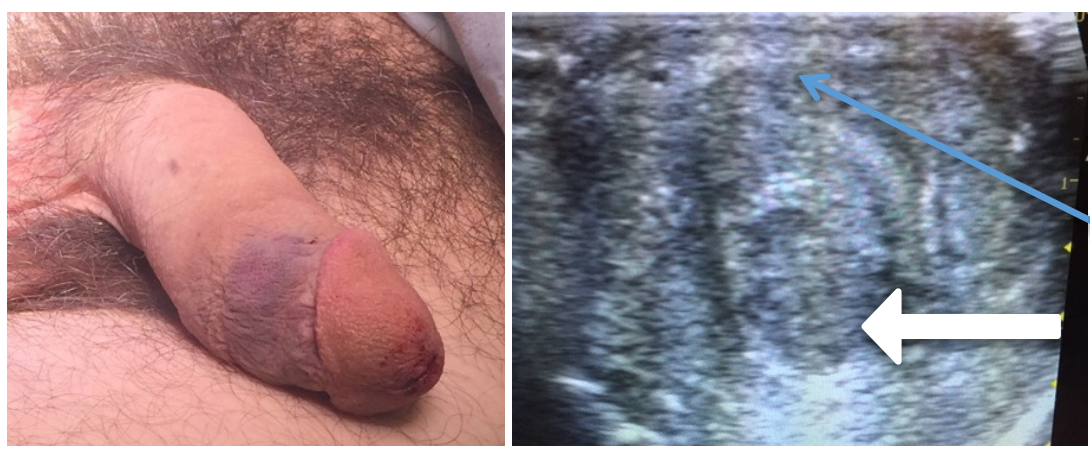

Tunica albuginea (blue arrow). Intracavernosal hematoma (white arrow).

Figure 5 A 47-year-old male presents after traumatic intercourse with a "pop" in his penis associated with severe penile pain and rapid detumescence 12 hours prior. He experienced significant urethral bleeding. Penile US revealed a right corporal intracavernosal hematoma (image is oriented with the dorsal TA in the superior aspect of the image and the urethra in the inferior aspect). Surgical exploration revealed right corporal rupture involving the urethra which was repaired surgically. The patient recovered full potency and voids without difficulty. US, ultrasound; TA, tunica albuginea. 

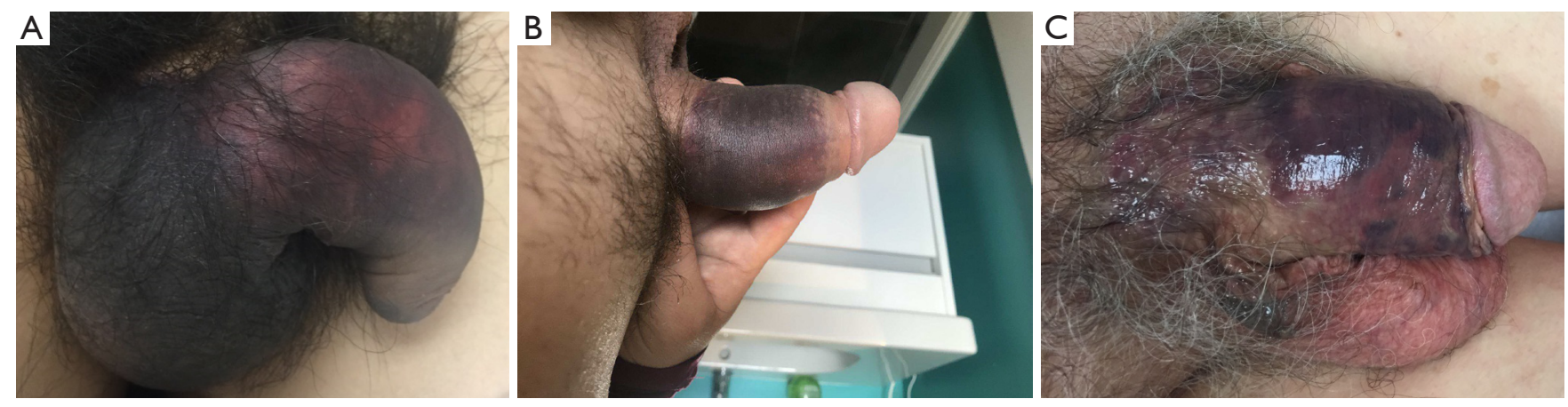

Figure 6 Varying degrees of post-CCH ecchymosis and edema. Intact tunica albuginea on penile ultrasound. (A) A 21-year-old male, 1 day after first $\mathrm{CCH}$ injection. Painless ecchymosis developed after nocturnal erection; (B) a 41-year-old male, 2 weeks after his first CCH cycle. Painless ecchymosis developed after receiving fellatio; (C) a 75 -year-old male, 3 weeks after his first $\mathrm{CCH}$ cycle. He presented to the emergency room after attempting intercourse and feeling a popping sensation with subsequent ecchymosis. CCH, collagenase clostridium histolyticum.

revealed a right mid-shaft $1 \mathrm{~cm}$ disruption of the corpora which was repaired surgically. His post-surgical erectile function is normal.

The circular intracavernosal hematoma within the circular TA creates a sonographic "Turkish eye sign" that indicates true penile fracture (Figure 5). Any patient with a history suggestive of fracture and penile ecchymosis associated with an intracavernosal hematoma should undergo penile exploration and surgical repair.

$\mathrm{CCH}$, approved for treatment of Peyronie's disease by the FDA in 2013, has revolutionized treatment for Peyronie's disease but confounded the diagnosis of penile ecchymosis and penile fracture. Injection of $\mathrm{CCH}$ into Peyronie's plaque leads to an enzymatic disruption of the collagen fibers that compose the abnormal plaques. Approval of $\mathrm{CCH}$ was based on the double-blinded, phasethree IMPRESS trials I and II (17). Over $84 \%$ of patients in the IMPRESS trials experienced an adverse event with the majority of these events $(80 \%)$ being minor penile ecchymosis, swelling, or pain. Penile hematoma or corporal rupture occurred in six patients $(0.01 \%)$, but the overall low rate of serious adverse events led to FDA approval. Since that time, both urologists and patients have enthusiastically embraced the use of $\mathrm{CCH}$ as one of the only truly effective non-surgical treatments for $\mathrm{PD}$.

In the new era of $\mathrm{CCH}$ treatment for $\mathrm{PD}$, severe penile ecchymosis or hematoma with or without corporal rupture is an increasingly common concern and proper diagnosis of this condition is essential for proper patient counseling and management. A recent survey of the Sexual Medicine Society of North America (SMSNA) reported that 64\% of respondents $(\mathrm{n}=100)$ had performed $>10 \mathrm{CCH}$ injections (18). Of survey respondents, $34 \%$ had encountered corporal rupture, and $67 \%$ managed fractures with penile exploration and repair (18). Diagnosis was by history/physical exam or additional imaging (MRI, US, or both) in $49 \%$ and $51 \%$ of patients, respectively. Sixty-two percent of the responders reported tissue quality worse than usually encountered in penile fracture cases. Interestingly, no significant difference was reported regarding erectile function, ability to have intercourse, or change in penile curvature after surveillance versus surgery, suggesting that perhaps "fracture" after $\mathrm{CCH}$ might have a different pathophysiology than the usual traumatic penile fracture. The only reported difference was in mean time to resumption of sexual intercourse (4.8 weeks for conservative vs. 7.2 weeks surgical management).

Often within 12-24 hours after injection, CCH patients may develop tremendous ecchymosis of the penile shaft, suprapubic region, and scrotal skin. It is also common that several weeks after injection, long after the recommended two-week period of abstinence is over, penile ecchymosis can recur and lead to concerns of possible penile fracture (Figure 6). These three patients display varying degrees of significant ecchymosis after $\mathrm{CCH}$ injection. All these patients had bedside sonograms revealing an intact TA, normal corpora cavernosa, and varying degrees of superficial soft tissue edema.

We theorize that there are likely two explanations for this phenomenon which are both likely in part responsible. As these events are most commonly associated with spontaneous penile erection and not sexual intercourse, it is most likely that penile erection causes radial and 
axial expansion of the attenuated PD plaque. This causes shearing or tearing of the superficial penile vessels which are now unsupported as the $\mathrm{CCH}$ has effectively attenuated the plaque and surrounding collagen adjacent to the vessels. An alternative, or perhaps complimentary, explanation is that "micro-fractures" of the plaque/TA develop after $\mathrm{CCH}$ which lead to the same clinical presentation as penile fracture but without "significant" tunical rupture or intracavernosal hematoma that can be visualized on US.

There are no clinical studies involving US or MRI in the diagnosis of a corporal fracture in post-CCH patients. There is one interesting case report of a false positive MRI in a post-Xiaflex patient that had subsequent exploration and was found to have intact TA (with a tear of Buck's fascia) (19). The authors concluded that interpretation of an MRI is made more challenging after $\mathrm{CCH}$ due to anatomic changes induced by $\mathrm{CCH}$ and thus the specificity of the MRI in these cases is decreased. This further supports our proposal that US is an effective tool to utilize in deciding to operate or not to operate.

Given the litigious nature of sexual medicine, it would be advantageous to have a consensus statement to guide urologists on the most appropriate workup and treatment of suspected penile fractures in the post-CCH patient (19). But, as $\mathrm{CCH}$ is a relatively new treatment modality for PD and most urologists are on the upswing of the learning curve, it will likely take many more years for clinical guidelines to be developed as expertise is developed through experience. This increasingly common scenario of post-CCH ecchymosis requires use of penile US to differentiate patients for whom emergent operative repair is indicated from those who can be managed conservatively. The US signs to inspect for are: (I) clear tunical rupture, which is most often absent; and (II) the presence of an intracavernous hematoma or the "Turkish eye sign". If neither of these signs is present, the urologist can be confident that observation is an appropriate option. If either or both of these signs is present, the patient should be surgically explored.

\section{Conclusions}

As demonstrated by the above cases, the combination of clinical history and physical examination plus penile US is a prudent approach to patients suspected of having a penile fracture. Physical exam frequently cannot differentiate a penile fracture from a superficial penile hematoma or penile ecchymosis. Penile US allows for clear imaging of the tunica and corpora cavernosa of the penis and can easily detect tunical disruption and intracavernosal hematoma ("Turkish eye sign"). If the TA is intact and the corpora appear homogenous, the risk of clinically significant penile fracture is very small. If the tunica is clearly disrupted, the patient should be surgically explored. If there is an isolated intracorporeal hematoma ("eye sign") on US, the patient has a corporal rupture and should be explored. If there is any doubt, an MRI can be obtained. If all imaging is equivocal, the choice for conservative management versus surgical exploration should be made through shared decision making with the patient with a low threshold for surgical exploration.

Even in cases where penile exploration and surgical repair is certain, preoperative penile US is a useful tool to help identify the specific location of fracture and facilitate placement of the surgical incision in the correct location. This avoids unnecessary penile degloving and its associated morbidity. The hematoma site does not always overlie the tunica injury as anatomical tissue planes allow for spread of the hematoma outward through the superficial soft tissue. Identification of the exact site of corporal rupture allows a small, targeted, longitudinal incision over the injury site (12).

Conservative treatment of patients with penile ecchymosis or hematoma and no penile fracture (intact TA and no intracorporeal hematoma, or "Turkish eye sign") involves ice, non-steroidal anti-inflammatory medications (NSAIDS) if no contraindications, and compression with a loosely-applied compressive dressing, replaced daily, for 1-2 weeks to prevent further bleeding from the ruptured vessels. We recommend against sexual activity for 4 weeks.

In the era of widespread $\mathrm{CCH}$, it is likely that penile ecchymosis, hematoma, and fracture presentations will become increasingly more common, and all practicing urologists who provide urgent care coverage should be comfortable with penile US as a modality for differentiating between penile ecchymosis, subcutaneous hematoma, and penile fracture to avoid unnecessary surgical exploration for these patients. At our institution, we have extensive experience with $\mathrm{CCH}$ injection and with management of post-injection complications. Thus far, we have not surgically explored for post-CCH ecchymosis or hematoma after over 1,650 injections, and all patients thus far have had preserved erectile function after conservative management as discussed.

\section{Acknowledgements}

None. 


\section{Footnote}

Conflicts of Interest: The authors have no conflicts of interest to declare.

\section{References}

1. McAninch JW, Santucci RA. Genitourinary Trauma. In: Walsh PC, Retick AB, Vaughan ED. et al. editors. Campbell's Urology (8th Edition). Philadelphia: Saunders, 2002:3707-44.

2. Beysel M, Tekin A, Gurdal M, et al. Evaluation and treatment of penile fractures: accuracy of clinical diagnosis and the value of corpus cavernosography. Urology 2002;60:492-6.

3. De Luca F, Garaffa G, Falcone M, et al. Functional outcomes following immediate repair of penile fracture: a tertiary referral centre experience with 76 consecutive patients. Scand J Urol 2017;51:170-5.

4. Koifman L, Barros R, Junior RA, et al. Penile fracture: diagnosis, treatment and outcomes of 150 patients. Urology 2010;76:1488-92.

5. Rosenstein DI, Morey AF, AmAninch JW. Penile Trauma. In: Graham SD, GLenn JF, Keane TE. editors. Glenn's Urologic Surgery (6th Edition). Philadelphia: Lippincott Williams \& Wilkins, 2004:582-7.

6. Guler I, Odev K, Kalkan H, et al. The value of magnetic resonance imaging in the diagnosis of penile fracture. Int Braz J Urol 2015;41:325-8.

7. Maurice MJ, Spirnak JP. The impracticality of MRI for the diagnosis of atypical penile fracture in the emergency setting. Emerg Med J 2014;31:42 1-2.

8. Mydlo JH, Hayyeri M, Macchia RJ. Urethrography and cavernosography imaging in a small series of penile fractures: a comparison with surgical findings. Urology 1998;51:616-9.

9. Fergany AF, Angermeier KW, Montague DK. Review of

Cite this article as: Metzler IS, Reed-Maldonado AB, Lue TF. Suspected penile fracture: to operate or not to operate? Transl Androl Urol 2017;6(5):981-986. doi: 10.21037/tau.2017.07.25
Cleveland Clinic experience with penile fracture. Urology 1999;54:352-5.

10. Kervancioglu S, Ozkur A, Bayram MM. Color Doppler sonographic findings in penile fracture. J Clin Ultrasound 2005;33:38-42.

11. El-Assmy A, El-Tholoth HS, Abou-El-Ghar ME, et al. False penile fracture: value of different diagnostic approaches and long-term outcome of conservative and surgical management. Urology 2010;75:1353-6.

12. Zare Mehrjardi M, Darabi M, Bagheri SM, et al. The role of ultrasound (US) and magnetic resonance imaging (MRI) in penile fracture mapping for modified surgical repair. Int Urol Nephrol 2017;49:937-45.

13. Mahapatra RS, Kundu AK, Pal DK. Penile Fracture: Our Experience in a Tertiary Care Hospital. World J Mens Health 2015;33:95-102.

14. Unnikrishnan R, Goel R, Thupili C, et al. Ultrasound of acute penile fracture. J Urol 2013;190:2253-4.

15. Shukla AK, Bhagavan BC, Sanjay SC, et al. Role of ultraosonography in grading of penile fractures. J Clin Diagn Res 2015;9:TC01-3.

16. Ash A, Miller J, Preston D. Point-of-care ultrasound used to exclude penile fracture. Crit Ultrasound J 2012;4:17.

17. Gelbard M, Goldstein I, Hellstrom WJ, et al. Clinical efficacy, safety and tolerability of collagenase clostridium histolyticum for the treatment of peyronie disease in 2 large double-blind, randomized, placebo controlled phase 3 studies. J Urol 2013;190:199-207.

18. Yafi FA, Anaissie J, Zurawin J, et al. Results of SMSNA Survey Regarding Complications Following Intralesional Injection Therapy With Collagenase Clostridium Histolyticum for Peyronie's Disease. J Sex Med 2016;13:684-9.

19. Beilan JA, Baumgarten AS, Bickell M, et al. Suspected Penile Fracture After Xiaflex Injection and Use of a VED. Urology 2016;98:4-7. 